\title{
UNDESCRIBED PLANTS FROM GUATEMALA AND OTHER CENTRAL AMERICAN REPUBLICS. XXXIII ${ }^{x}$
}

\author{
JoHN DONNELL SMITH
}

Anona (§AtTaE Mart.) macroprophyllata Donn. Sm.-Folia subsessilia parva elliptico- vel obovato-oblonga apice rotundata vel saltem obtusa basi rotundata vel retusa. Pedunculi laterales ad basin bracteis binis foliaceis cordato-orbicularibus fulti. Petala exteriora oblonga obtusa, interiora deficientia.

Frutex $3-4$-metralis. Folia pergamentacea glabra $4-5 \cdot 5^{\mathrm{cm}}$ longa $2-3^{\mathrm{cm}}$ lata 7-9-penninervia minutissime reticulata pellucido-punctulata, petiolis $2-3^{\mathrm{mm}}$ longis. Pedunculi glabri $23^{-2} 7^{\mathrm{mm}}$ longi, bracteis suboppositis primum ferrugineosericeis denique praeter basin marginemque ciliatas glabrescentibus, inferiore $20-24^{\mathrm{mm}}$-diametrali quam altera subdimidio majore, bracteola ad medium pedunculi minuta lanceolata sericea. Sepala ovata $3-4^{\mathrm{mm}}$ longa extus ferrugineovillosa. Petala extus glabrescentia intus cinereo-velutina $2 \mathrm{I}^{\mathrm{mm}}$ longa $8^{\mathrm{mm}}$ lata basi concava. Stamina $2.5^{\mathrm{mm}}$ longa puberula, filamentis antheras subaequantibus, connectivo ultra loculos pulvinato. Torus staminiferus pilosus. Pistilla $2^{\mathrm{mm}}$ longa. Fructus desideratur.

In praecipite prope Fiscal, Guatemala, alt. I1 10m, Jun. 1909, Charles $C$. Deam n. 6rgI.-Typus in herbario Musei Nationalis servatur.

Krameria (\$Tetrandrae Chod.) dichrosepala Donn. Sm.Cano-sericea. Folia lanceolato-linearia. Pedunculi axillares folia subaequantes supra medium bibracteolati. Sepala 4, duobus intermediis et petalis flavicantibus. Petala superiora sepalis paulo breviora unguibus totis coalita, laminis flabelliformibus. Stamina infra medium unguibus petalorum adnata.

Fruticulus prostratus, ramis virgatis 3 -5-decimetralibus cum foliis et pedunculis cano-sericeis hinc illinc rufescentibus. Folia sessilia $7-9^{\mathrm{mm}}$ longa $\mathrm{I}-2^{\mathrm{mm}}$ lata in mucronem nigrum $0.5^{\mathrm{mm}}$ longum attenuata basi rotundata margine revoluta. Pedunculi $5^{-8^{\mathrm{mm}}}$ longi, bracteis foliaceis $5^{-6^{\mathrm{mm}}}$ longis. Sepala parum inaequalia $7-8^{\mathrm{mm}}$ longa oblongo-ovata obsolete mucronata extus sericea, extimo valde gibboso et intimo sanguineis, intermediis inaequilateralibus. Petala superiora $6^{\mathrm{mm}}$ longa, laminis $\mathrm{I} .5^{\mathrm{mm}}$ longis $2^{\mathrm{mm}}$ latis subcrenulatis, intermedio paulo minore, petalis inferioribus suborbicularibus $2^{\mathrm{mm}}$-diametralibus subdenticulatis glabris. Stamina $4.5^{\mathrm{mm}}$ longa. Ovarium gibbosum ovale cano-villosum addito I Continued from Bot. Gazette 48:300. I909. 
stylo aequilongo stamina paulo superans. Fructus ignotus.-Ad $K$. canescentem A. Gray et $K$. bicolorem S. Wats. indumento foliis inflorescentia accedens ab utraque inter alia petalis staminibusque connatis recedit.

Ad viam montanam flumen Montagua ultra hand procul a Gualán, Depart. Zacapa, Guatemala, Jun. I909, Charles C. Deam n. 6273.-Typus in herb. Musei Nationalis servatur.

Calliandra (\$MACRophyllaE Benth.) rhodocephala Donn. Sm. - Glabra. Folia breviter petiolata, pinnis unijugis, foliolis trijugis, addito saepius foliolo infimo solitario, inaequilateralibus oblongovel ovato-lanceolatis acute acuminatis basi exteriore rotundatis interiore acutis binerviis. Pedunculi axillares et terminales fasciculati breves. Calyx striatus et corolla erubescens.

Frutex 2.5-metralis. Stipulae ramentaceae oblongo-ovatae $8^{\mathrm{mm}}$ longae persistentes. Petiolus communis $\mathbf{I}-\mathbf{I} \cdot 5^{\mathrm{cm}}$ longus, pinnarum rhachi $7-\mathbf{r} .05^{\mathrm{cm}}$ longa, foliolis supra praeter nervos puberulos vernicosis in jugo inaequalibus per paria deorsum decrescentibus, terminalibus $10-12^{\mathrm{cm}}$ longis $3 \cdot 5-4 \cdot 2^{\mathrm{cm}}$ latis, in pare infimo $3 \cdot 5^{-5} \cdot 5^{\mathrm{cm}}$ longis, foliolo septimo $2-2 \cdot 5^{\mathrm{cm}}$ longo. Pedunculi circiter $\mathrm{I} \cdot 5^{\mathrm{cm}}$ longi basi bracteis striatis ovato-lanceolatis $8^{\mathrm{mm}}$ longis medio bracteola minuta muniti, capitulo glabro, juvenili etiam in sicco laete rosaceo, bracteolis floralibus ovalibus $1.5^{\mathrm{mm}}$ longis striatis nigro-apiculatis. Calyx obconicus $3^{\mathrm{mm}}$ longus, lobis rotun- datis $0.5^{\mathrm{mm}}$ longis apice nigris. Corolla $8^{\mathrm{mm}}$ longa supra dimidiam tubulosam infundibuliformis, lobis triangularibus $2^{\mathrm{mm}}$ longis trigono-incrassatis. Stamina rosacea $2 \cdot 5^{\mathrm{cm}}$ longa, tubo breviter exserto, antheris glanduloso-puberulis. Legumen ignotum.-C. bolivianae Britton proxima.

Prope viae ferreae pontem ad Puerto Barrios, Depart. Yzabal, Guatemala, Maj. r909, Charles C. Deam n. 6015.-Typum in herb. Musei Nationalis vidi.

Casearia (§Píumba Benth.; Warburg) nicoyensis Donn. Sm.Folia lanceolato-elliptica obtuse acuminata basi acuta serrulata supra glabra subtus glabrescentia, punctis atque lineis obsoletis. Pedicelli fasciculati petiolum subaequantes bracteis majusculis bis longiores flore subbreviores. Calycis segmenta linearia. Stamina 8 glabra breviter connata, filamentis staminodia pilosa paulo superantibus.

Inermis, ramulis novellis stipulis foliorum nascentium tergo pedicellis calyce sordide pubescentibus. Folia coriacea $5 \cdot 5-8.5^{\mathrm{cm}}$ longa medio $2 \cdot 5^{-} 3^{\mathrm{cm}}$ lata minute appresseque calloso-serrulata erga lucem inspecta pellucido-reticulata, petiolis $3^{-5^{\mathrm{mm}}}$ longis, stipulis lanceolato-ovatis $2^{\mathrm{mm}}$ longis. Bracteae ad nodos foliatos numerosae imbricatae ovatae $2^{\mathrm{mm}}$ longae rufidulo-membranaceae glabrescentes, pedicellis $3 \cdot 5-4^{\mathrm{mm}}$ longis. Calycis $5^{\mathrm{mm}}$ longi segmenta paene sejuncta $\mathrm{I}^{\mathrm{mm}}$ lata obtusa intus glabra. Discus staminiferus $\mathrm{I}^{\mathrm{mm}}$ altus, filamentis $2^{\mathrm{mm}}$ 
longis, antheris oblongo-ellipsoideis $0.5^{\mathrm{mm}}$ longis nudis, staminodiis $\mathrm{I} \cdot 5^{\mathrm{mm}}$ longis totis patenter cano-pilosis. Ovarium pubescens ellipsoideum $1.5^{\mathrm{mm}}$ longum in stylum aequilongum indivisum attenuatum, placentis tribus. Capsula deficit.

In fruticetis et secus vias, Peninsula Nicoya, Costa Rica, Apr. I900, Adrian Tonduz n. I390I.

Reynoldsia americana Donn. Sm. Folia ter pinnata, foliolis bijugis cum impare lanceolato-ovatis glandulari-serratis. Umbellae subcapituliformes multiflorae racemosae, racemis fasciculatis pedunculatis, floribus 9-Io-meris. Corolla clausa ovato-globosa, petalis oblongis.

Arbor mediocris. Ramuli fistulosi foliorum cicatricibus approximatis notati. Folia juvenilia solum visa petiolo 8-I $\mathrm{I}^{\mathrm{cm}}$ longo computato $27-30^{\mathrm{cm}}$ longa, pinnis primariis 4-jugis, secundariis 3 -jugis, foliolis $3 \cdot 5^{\mathrm{cm}}$ longis $2^{\mathrm{cm}}$ latis, petiolo dilatatoamplexicauli, stipulis obsoletis. Pedunculi circiter 6 -fasciculati $2 \cdot 5-3 \cdot 5^{\mathrm{cm}}$ longi cum racemi rhachi $\mathrm{I} \cdot 5^{-2^{\mathrm{cm}}}$ longa crassi, ramis $3-4^{\mathrm{cm}}$ longis plerumque ad apicem rhacheos 5-8-fasciculatis, umbellis subglobosis 22-36-floris, pedicellis teretibus $5^{-6 \mathrm{~mm}}$ longis inarticulatis, bracteis bracteolisque ovatis parvis, floribus praecocibus ecalyculatis $4^{\mathrm{mm}}$ longis. Calyx hemisphaericus $3^{\mathrm{mm}}$ latus, limbo integro glandulis circiter 6 apiculato. Petala 9-Io calyptratim cohaerentia et sub anthesi dejecta $3 \cdot 5^{\mathrm{mm}}$ longa $\mathrm{I}^{\mathrm{mm}}$ lata apice angustata. Stamina 9-Io, filamentis superne inflexis, antheris oblongis $2^{\mathrm{mm}}$ longis. Stigma 9-Io-radiatum stylopodio conico impositum. Drupa ignota.-Species hactenus cognitae insularum Oceani Pacifici incolae sunt.

In silvis, Peninsula Nicoya, Costa Rica, Jan. I900, Adolfo Tonduz n. I3823.Nomen vernaculare Chile.-Typus in herb. Musei Nationalis servatur.

Bouvardia Deamii Donn.Sm.-Folia opposita rotundo- vel lanceolato-ovata obtuse acuminata basi rotundata vel acutiuscula. Stipulae integrae deltoideae mucronatae. Pedunculus terminalis $3-5$-florus interdum in axilla utraque paenultima uniflorus. Corollae limbus intus cano-velutinus, lobi suborbiculares.

Frutex orgyalis, ramulis divaricatis, novellis sicut stipulae petioli folia nascentia inflorescentia puberulis. Folia supra glabrescentia subtus nervis puberula concoloria $33^{-} 3^{8 \mathrm{~mm}}$ longa $15^{-2} 5^{\mathrm{mm}}$ lata, nervis lateralibus utrinque $3-4$, petiolis $\mathrm{I}^{\mathrm{mm}}$ longis, stipulis $\mathrm{I} \cdot 5^{\mathrm{mm}}$ longis. Pedunculus saepius triflorus $2.3^{\mathrm{mm}}$ longus, pedicellis bracteolisque $\mathbf{I} \cdot 5^{-2^{\mathrm{mm}}}$ longis. Calycis tubus velutinus globosus I $.5^{-}$ $2^{\mathrm{mm}}$-diametralis lobos subulatos $2-3$-plo superans. Corollae tubus cylindricus $9^{-10^{\mathrm{mm}}}$ longus rufescens extus sparsim puberulus intus glaber faucibus staminiferis ampliatus ore constrictus, lobi late patentes $3-3 \cdot 5^{\mathrm{mm}}$ longi carnulosi intensius coccinei extus glabri. Filamenta tubo ad $2^{\mathrm{mm}}$ infra os breviter adnata, antheris subsessilibus basifixis $2^{\mathrm{mm}}$ longis. Discus glaber. Stylus glaber $4^{\mathrm{mm}}$ longus, 
stigmatis bipartiti ramis $\mathrm{I} .5^{\mathrm{mm}}$ longis. Placentae ovula involventes. Capsula non adest.

Ad fundum praecipitii prope Fiscal, Guatemala, alt. I I Iom, Jun. 1909, Charles C. Deam n. 6rgo.-Typus in herb. Musei Nationalis servatur.

Tonduzia Pittieri Donn. Sm.-Folia 3-4-natim verticillata membranacea lanceolato-elliptica 3-4-plo longiora quam latiora. Calycis segmenta acuta membranacea margine hyalina. Corolla ore canopilosa ceteroquin glabra tubo medio staminifero dilatata. Discus obsoletus. Folliculi divaricati.

Arbuscula 3.5-4.5-metralis omnibus in partibus excepto corollae ore glaberrima. Folia cujusque paris inaequalia $8-12^{\mathrm{cm}}$ longa $3-3 \cdot 5^{\mathrm{cm}}$ lata utrinque praesertim superne acuminata nitida subtus pallidiora pellucido-nervata et punctulata, superiora ternata, nervis lateralibus sub margine arcuatis, interjecto interdum altero breviore, venis obsoletis, petiolis $6-9^{\mathrm{mm}}$ longis. Cyma pseudoterminalis corymbiformis laxe ramosa resinoso-glandulosa $5-7^{\mathrm{cm}}$ alta $9^{-10^{\mathrm{cm}}}$ lata, bracteolis membranaceis lanceolato-ovatis acutis, pedicellis $3-5^{\mathrm{mm}}$ longis. Calyx $\mathrm{I} \cdot 5^{\mathrm{mm}}$ longus, segmentis ovatis aegre $\mathrm{I}^{\mathrm{mm}}$ longis. Corollae totae $\mathrm{I}^{\mathrm{cm}}$ longae segmenta tubum aequantia oblongo-ovalia bis longiora quam latiora apice rotundata tenuiter membrancea venosa. Stamina glabra, antheris subsessilibus. Ovarii carpella semiconnata, stylo basi vix fisso $\mathrm{I}^{\mathrm{mm}}$ longo uti stigma $0.5^{\mathrm{mm}}$ longum glabro, ovulis in utraque serie circiter 6 . Folliculi $6.5^{-} 7^{\mathrm{cm}}$ longi $5^{\mathrm{mm}}$ crassi. Semina ignota.- Hanc ab utraque specie hucusque cognita foliis pro rata latioribus facile dignoscendam claro $H$. Pittier, TondUZIAE auctori, dicavi.

In praecipite ad Fiscal, Guatemala, alt. II Iom, Jun. I909, Charles C. Deam n. 6og8. - In silvis prope Gualán, Depart. Zacapa, Guatemala, alt. I86 ${ }^{\mathrm{m}}$, Jun. I909, Charles C. Deam n. 6307.-Typos in herb. Musei Nationalis servatos vidi.

Marsdenia gualanensis Donn. Sm.-Folia utrinque puberula punctata orbiculari-cordata cuspidata 5-nervia. Paniculae axillares sessiles vel breviter pedunculatae petiolum subaequan tes inferne bis terve furcatae, ramis suberectis incrassatis, pedicellis gracilibus. Corolla extrorsum glabra, faucibus biseriatim cano-barbatis.

Frutex volubilis 3-metralis, ramis petiolisque glabrescentibus. Folia membranacea in cuspidem $\mathbf{I}-\mathbf{I} .5^{\mathrm{cm}}$ longam acutam mucrunculatam subabrupte acuminata absque cuspide $6-8^{\mathrm{cm}}$ longa atque lata, sinu rotundato, ima basi cumeata supra glanduloso-maculata, nervis lateralibus praeter basales utrinsecus tribus, venis subtransversalibus, venulis reticulatis pellucidis, petiolis $2.5-3^{\mathrm{cm}}$ longis. Paniculae pubescentes $3-3 \cdot 5^{\mathrm{cm}}$ longae, ramis bracteosis $12-18^{\mathrm{mm}}$ longis, pedicellis $4^{-} 7^{\mathrm{mm}}$ longis. Calyx pubescens $2^{\mathrm{mm}}$ longus, segmentis oblongo-ovatis obtusis, sinubus eglandulosis. Corollae tubus campanulatus lobos obtuse ovatos atque calycem aequans. Coronae squamae oblongo-ellipticae $0.5^{\mathrm{mm}}$ longae gynostegio 
dimidio breviores obtusae superne hyalinae. Discus stylinus $0.5^{\mathrm{mm}}$-diametralis breviter rostellatus. Carpidia glabra ovoidea $0.5^{\mathrm{mm}}$ longa. Folliculi desunt.

In fruticetis ad viam prope Gualán, Depart. Zacapa, Guatemala, alt. $186^{\mathrm{m}}$, Jun. 1909, Charles C. Deam n. 6333.-Specimen typicum in herb. Musei Nationalis servatur.

\section{Heliotropium ( $\$$ ORThostachys A.DC.) physocalycium Donn.} Sm.-Omnibus fere in partibus glanduliferum. Folia supra scabridiuscula sparsim strigillosa subtus molliter pilosa lanceolato-oblonga trảnsversim venosa. Pedunculi laterales et terminales dichotomi. Calyx fructiferus auctus inflatus. Stylus brevissimus. Fructus oblato-globosus, nucibus dorso exsculpato-reticulatis.

Frutex 3-metralis. Rami petioli pedunculi spicae necnon calyces simul pubescentes et pilis longis patentibus glandularibus conspersi. Folia $8-I I .5^{\mathrm{cm}}$ longa $2 \cdot 5^{\mathrm{cm}}$ lata utrinque attenuata, petiolis $8-15^{\mathrm{mm}}$ longis. Pedunculi bis terve dichotomi $5^{-} 8^{\mathrm{cm}}$ longi, spicis $7-14^{\mathrm{cm}}$ longis, junioribus scorpioidibus, bracteis bracteolisque obsoletis. Calycis floriferi $3^{\mathrm{mm}}$ longi segmenta lanceolatoovata herbacea nervosa, fructiferi pentagono-globosi segmenta sibi invicem marginibus applicata late ovata cuspidato-acuminata $4^{\mathrm{mm}}$ longa. Corollae tubus 4-4. $5^{\mathrm{mm}}$ longus extus glandulari-puberulus intus glaber ad $\mathrm{I} .5^{\mathrm{mm}}$ supra basin staminiferus, lobi semiorbiculares $\mathrm{I}^{\mathrm{mm}}$ longi, sinubus nudis. Antherae vix cohaerentes lineari-oblongae $\mathrm{I} .5^{\mathrm{mm}}$ longae apice papillosae. Stigma $\mathrm{I}^{\mathrm{mm}}$ longum stylo paulo longius, apice truncato-conico glabro. Fructus glandulari-puberulus I. $5^{\mathrm{mm}}$ altus $2^{\mathrm{mm}}$ latus profunde 4 -sulcatus, nucibus leviter cohaerentibus, seminibus rectis.

Capetillo, Depart. Zacatepéquez, Guatemala, alt. I400 ${ }^{\mathrm{m}}$, Mart. I892, John Donnell-Smith n. 2472 ex Pl. Guat. etc. quas ed. Donn. Sm.-Laguna de Ayarza, Depart. Jalapa, Guatemala, alt. 2400 ${ }^{\mathrm{m}}$, Sept. I892, Heyde et Lux, no. 3990 ex Pl. Guat. etc. quas ed. Donn. Sm.-Guatemala, Depart. Guatemala,

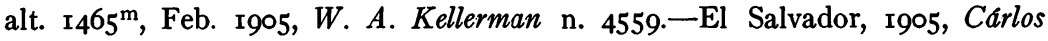
Rénson n. 258.-(Exemplum utrumque nempe Kellermanianum et Rensonianum in herb. Musei Nationalis vidi.)

Eandem plantam in Ecuador collegit et sub numeris $1443^{a}$ et $1485^{\circ}$ distribuit cl. Eggers.

Blechum (\$Eublechum Oerst.) pedunculatum Donn. Sm.Folia ovata in petiolum decurrentia. Pedunculi axillares spicam ovoideam subaequantes vel superantes, bracteis obovatis 3-5-nerviis unifloris. Corollae lobi obovati tubo longiores. Capsula tetrasperma, seminibus barbatis.

Fruticulus ascendens 24-30-centimetralis, ramis bifariam pubescentibus. Folia utrinque sparsim bulboso-strigillosa subtus nervis patenter pubescentia $3 \cdot 5^{-} 5^{\mathrm{cm}}$ 
longa $2-3^{\mathrm{cm}}$ lata obtuse acuminata ima basi in petiolum totum alatum I-I. $5^{\mathrm{cm}}$ longum abrupte contracta. Pedunculi pubescentes $\mathrm{I}-2^{\mathrm{cm}}$ longi, spica II-I $7^{\mathrm{mm}}$ longa, axe $6-9^{\mathrm{mm}}$ longo, bracteis circiter ro- 16 apice rotundatis basi attenuatis ciliatis $7-\mathrm{II}^{\mathrm{mm}}$ longis $4^{-8^{\mathrm{mm}}}$ latis, bracteolis linearibus $\mathrm{I}^{\mathrm{mm}}$ longis hyalinis. Calycis segmenta lineari-lanceolata $3^{\mathrm{mm}}$ longa scariosa nervata. Corollae tubus rectus superne angustatus $5^{\mathrm{mm}}$ longus, lobi inaequales, extimus maximus $8^{\mathrm{mm}}$ longus atque latus extus pubescens basi intus aurantiaceus. Stamina summo tubo inserta, antheris subsessilibus. Stylus $3^{\mathrm{mm}}$ longus. Capsula ellipticooblonga $7^{\mathrm{mm}}$ longa supra medium vacua. Semina pilis rectis glandularibus scabridiusculis munita, retinaculis lanceolato-dilatatis.

Ad viam montanam prope Gualán, Depart. Zacapa, Guatemala, alt. $186^{\mathrm{m}}$, Jun. 1909, Charles C. Deam n. 6277.-Typus in herb. Musei Nationalis servatur.

Baltimore, Maryland 